\title{
Epidemiology of long-stay patients in the pediatric intensive care unit: prevalence, characteristics, resource consumption and complications
}

\author{
S. Miura ${ }^{1} \cdot$ M. Fukushima ${ }^{1} \cdot$ H. Kurosawa ${ }^{1} \cdot$ S. Kimura ${ }^{1}$ \\ Received: 2 December 2019 / Accepted: 1 April 2020 / Published online: 17 April 2020 \\ (C) Springer-Verlag GmbH Germany, part of Springer Nature 2020
}

\begin{abstract}
Background The impact of pediatric intensive care unit (PICU) utilization and resource consumption among long-stay patients has not been characterized recently. This study aimed to describe the resource consumption and characteristics of long-stay patients in a PICU.

Methods This was a single-center descriptive cohort study of 1309 patients admitted to a PICU in 2017. The main outcome was ICU length of stay (LOS). Patients were divided into prolonged LOS (PLS) and non-PLS groups if they had an LOS of $\geq 28$ or $<28$ days, respectively. Two groups were compared to characterize PLS.

Results Thirty-two (2.4\%) patients had a PLS and utilized 33\% of PICU bed days. Factors associated with PLS with odds ratio [95\% confidence interval (CI)] were being a neonate (7.8 [2.5-25.4], $p=<0.001)$, being an infant (2.9 [1.0-9.0], $p=0.04)$, admission for a respiratory ailment (7.3 [1.6-44.2], $p=0.003)$, cardiovascular dysfunction $(24.1$ [4.8-152.1], $p=<0.001)$, postcardiac operation (8.0 [1.7-50.1], $p=0.003)$, post-cardiopulmonary arrest (22.8 [1.7-211.9], $p=0.01)$, and transfer from another facility $(4.2[1.8-10.7], p=0.001)$. PLS patients developed more nosocomial infections and disproportionately received monitoring and therapeutic resources.

Conclusions A PLS was associated with substantial PICU utilization and complication rates. Future studies should aim to alleviate both institutional and patient-related issues in the affected population harboring possible risk factors for PLS.
\end{abstract}

Keywords Complication $\cdot$ Long stay $\cdot$ Pediatric intensive care $\cdot$ Resource utilization $\cdot$ Risk factor

\section{Introduction}

Although few patients require a prolonged length of stay (PLS) in an intensive care unit (ICU), these patients account for considerable numbers of ICU bed days (Pollack et al. 1987; Namachivayam et al. 2012; Marcin et al. 2001). In pediatric ICU (PICU) settings, PLS is considered a critical issue because the affected children face higher risks of comorbidities and mortality and a poor quality of life while consuming substantial patient care and financial resources (Pollack et al. 1987; Namachivayam et al. 2012; Marcin et al. 2001; Gonzalez-Cortes et al. 2011). Therefore, it is imperative to

S. Miura

shin.nya1982@gmail.com

1 Department of Pediatric Intensive Care, Saitama Children's Medical Center, 1-2, Shintoshin, Saitama, Chuou-ku 330-8777, Japan balance the needs of patients in this population with available resources to optimize the efficiency and quality of critical care. In the literature, the reported prevalence of long-stay patients varies because of differences in the lengths of stay (LOSs), which may differ because of variations in factors such as illness severity, diagnostic diversity, and institutional practices (Straney et al. 2010; Ruttimann et al. 2000; Ruttimann and Pollack 1996). Additionally, these patients tend to receive complicated long-term care in the ICU (Namachivayam et al. 2012).

Evidence increasingly suggests that LOS can be reduced by the implementation of quality improvement programs such as fast-track care, weaning protocols for ventilated patients, early rehabilitation, nighttime extubation, and ICU specialization (Vricella et al. 2000; Reismann et al. 2012; Yamasaki et al. 2011; Tischenkel et al. 2016; da Silva et al. 2016; Ruttimann et al. 2000; Johnson et al. 2018; Dreyfus et al. 2017; Riker et al. 2009; McWilliams et al. 2015; Balas et al. 2013). However, the rates of PICU utilization and resource 
consumption among long-stay patients have not been characterized recently, and research is needed to describe the current statuses and effects of patients with a PLS. Previous studies have reported that predictors of a PLS include younger age, chronic illness, and emergent admission (Pollack et al. 1987; Straney et al. 2010; Marcin et al. 2001; Ruttimann and Pollack 1996). Nevertheless, many factors remain controversial, including the admission source, admission category (cardiac versus non-cardiac), and severity of the condition (Marcin et al. 2001; Ruttimann and Pollack 1996). Additionally, although previous long-term follow-up studies have reported a poor quality of life and increased mortality among long-stay patients (Pollack, et al. 1987; Namachivayam et al. 2012; Marcin et al. 2001), few have addressed whether long-stay patients face an increased risk of infectious complications during a PICU stay (Gonzalez-Cortes et al. 2011).

To address the limitations of previous research, we conducted this study to describe the prevalence, characteristics, resource consumption, and infectious complications of children with a PLS who were admitted to a combined medicalsurgical multidisciplinary-care PICU at a tertiary center following the implementation of fast-track care.

\section{Methods}

\section{Study patients}

This study included all patients aged $\leq 18$ years who were admitted to the PICU at Saitama Children's Medical Center in Japan from January 1, 2017, to December 31, 2017. Only the first admission for each patient during the study period was considered eligible for data analysis.

\section{Setting}

This PICU is a 34-bed combined medical-surgical, multidisciplinary-care tertiary care unit that serves children living in Saitama Prefecture, which has a pediatric population of 1.2 million. Each year, this PICU accommodates approximately 1600 admissions with diverse diagnoses and provides intensive care via a multi-disciplinary team. During the study period, discharge from the PICU was discussed during daily morning rounds, and final decisions were made by the attending intensivists. Indications for PICU admission included airway obstruction, acute respiratory failure potentially requiring respiratory support [e.g., humidified high-flow nasal cannula, non-invasive positive pressure ventilation, and mechanical ventilation (MV)], cardiovascular dysfunction, a deteriorated level of consciousness, and a requirement for heavy nursing care and meticulous monitoring. PICU patients were transferred to wards or other hospitals when relevant indications were addressed. The operation room (OR) was the most common origin of admission. Extubation in the OR was considered a standard practice unless the patient was at risk of extubation failure.

\section{Data collection and variable definitions}

The LOS in the PICU was recorded in hours and converted to days. We defined a PLS as an LOS of $\geq 28$ days and accordingly stratified patients into PLS ( $\geq 28$ days) and non-PLS ( $\leq$ 27 days) groups to ensure that our data were compatible with those of previous studies (Naghib et al. 2010; GonzalezCortes et al. 2011; Namachivayam et al. 2012). As the definition of PLS varies from 3 to 30 days in the literature (Marcin et al. 2001; Ruttimann and Pollack 1996; Pollack et al. 1987; Mahesh et al. 2012), we also conducted descriptive analyses using binned groups stratified by different PICU LOS as follows: $<3,3-6,7-13,14-27$, and $\geq 28$ days.

Factors that might influence LOS were identified from the literature a priori. For this study, the following variables were collected via chart reviews: age at admission, sex, LOS in the PICU, primary reason for admission, origin of admission, comorbidities, condition at admission, Pediatric Risk of Mortality-2 (PIM-2) score (Shann et al. 1997), devices and treatments used, complications, and ICU mortality. Patients were divided into eight categories according to the primary reason for admission, namely respiratory dysfunction, cardiovascular dysfunction, neurologic problem, postcardiopulmonary arrest, trauma, other medical condition, post-surgical cardiac patient, and post-surgical non-cardiac patient.

Comorbidities were classified into 11 major categories using the International Classification of Diseases, Tenth Revision, Clinical Modification. Up to two categories were recorded per patient, including diseases of the circulatory system, diseases of the respiratory system, diseases of the digestive system, diseases of the genitourinary system, diseases of the nervous system, hematology/immunodeficiency, neoplasms, endocrine/nutritional/metabolic disease, mental/behavior/neurodevelopmental disorders, congenital malformation or chromosomal abnormalities, and others (Namachivayam et al. 2012). The device variables included an arterial line, central venous line, and Foley catheter. Treatment-related variables included MV, vasoactive agent, renal replacement therapy (RRT), and extracorporeal membrane oxygenation (ECMO). Other conditions included chromosomal abnormality, pediatric cerebral performance category (PCPC) at ICU admission, emergent admission, use of MV within the first hour of admission, and dependency on total parenteral nutrition (TPN)/tube feeding/chronic respiratory support. Recorded complications and surgical interventions included ventilator-associated pneumonia (VAP), catheterrelated bloodstream infection (CRBSI), catheter-acquired urinary tract infection (CAUTI), cardiopulmonary arrest during 
the ICU stay, and need for a tracheostomy or gastrostomy. Infectious complications were recorded if clinically diagnosed and treated with antibiotics for a scheduled duration.

\section{Statistical analysis}

Subject data were censored at the time of PICU discharge or 180 days after the date of PICU admission. The proportions of long-stay patients and PICU bed days were calculated, and factors associated with a PLS were determined through univariate analyses. The chi-square test or Fisher's exact test was applied to categorical variables, while the Mann-Whitney U test was used for continuous variables. Patients aged $>$ 16 years were excluded from the analysis of PIM-2. To analyze time-varying variables such as infectious complications, survival outcomes were compared with respect to PLS versus non-PLS among patients using devices that carry a risk of nosocomial infection (e.g., central venous line, Foley catheter, endotracheal tube) using a log-rank test. Two-tailed $P$ values $<$ 0.05 were considered statistically significant. STATA 14 software (StataCorp LLC, College Station, TX, USA) was used for all statistical analyses.

\section{Results}

During the study period, 1618 episodes of admission and 7562 bed days were recorded for 1309 patients. After excluding 309 non-first admissions during the study period, 1309 admissions were finally included in the analysis. Among these cases, $44.1 \%$ of patients were female, $26.1 \%$ were younger than 1 year old, and $48.1 \%$ had at least one comorbidity. Medical and surgical admissions accounted for $56.8 \%$ and $43.2 \%$ of cases, respectively. The primary organ dysfunction was respiratory, cardiovascular, and neurologic in $11.4 \%$, $3.3 \%$, and $13.4 \%$ of cases, respectively, with $1.1 \%$ of patients being admitted post-cardiopulmonary arrest. Postoperative admission was the most common origin $(45.5 \%)$, followed by admission via the emergency room (29.4\%) and transfers from other hospitals $(22.8 \%)$ and wards $(2.2 \%)$. The ICU mortality rate was $1.3 \%$ (Table 1 ).

The median LOS for overall admissions was 1.6 days [interquartile range (IQR): 0.9-3.8 days], with a mean LOS of 4.5 days. Among patients with a PLS, the median LOS was 44.1 days (IQR: $35.9-76.8$ days). The median LOS of patients with a non-PLS was 1.5 days (IQR: 0.9-3.6 days). After stratification, 908 (69.4\%), 232 (17.7\%), 92 (7.0\%), 45 (3.4\%), and $32(2.4 \%)$ patients had an LOS of $<3,3-6,7-13,14-27$, or $\geq 28$ days, respectively. Of the last group ( $\geq 28$ days), $2.4 \%$ of patients accounted for $33.0 \%$ of overall ICU bed days. Additionally, the combined $5.9 \%$ of patients with an LOS $\geq$ 28 or 14-27 days accounted for $47 \%$ of bed days. By contrast, patients with an LOS of $<3$ and 3-6 days accounted for only $19.3 \%$ and $17.9 \%$ of ICU bed days, respectively (Fig. 1).

The results of univariate analysis by category are presented in Table 1 . Neonates ( $\leq 30$ days old) and infants were significantly more likely than school-aged children to have a PLS $(P<0.001$ and 0.04 , respectively). Additionally, patients admitted for respiratory dysfunction, cardiovascular dysfunction, post-cardiopulmonary arrest, and post-cardiac surgery had a significantly higher risk of PLS relative to those admitted post non-cardiac surgery $(P=0.003,<0.001,0.01$, and 0.003 , respectively), and those transferred from other hospitals had a higher likelihood of PLS relative to postoperative patients (Table 1). These findings were compatible with the trends observed in descriptive analyses stratified by the five LOS groups (Figs. 4, 5, 6).

Univariate analysis also revealed several variables associated with PLS, including a PCPC $\geq 2(P=0.01)$, multiple baseline comorbidities $(P=0.03)$, dependency on tube feeding $(P=0.004)$, emergent admission $(P=0.046)$, and MV within the first hour of admission $(P<0.001)$. By contrast, a PLS was not significantly associated with chromosomal abnormality, dependency on TPN, or chronic respiratory support (Table 1). Among patients aged $<16$ years, PIM-2-predicted mortality was associated with a PLS $(P=0.03)$, and descriptive analysis revealed a trend toward a positive association between a PIM-2 of 0-6\% with LOS. Notably, this trend inverted when PIM-2 exceeded 6\% (Fig. 3).

Additional analyses of device utilization found that therapeutic resources were more commonly used and surgical interventions applied to patients with a PLS (Table 2). The descriptive analysis of patients stratified by LOS found that the frequency of use of resources such as an arterial line, central venous line, Foley catheter, vasoactive drug, and MV increased with increasing LOS. Additionally, RRT, ECMO, tracheostomy, and gastrostomy were predominantly applied to patients with a PLS (Fig. 2). Finally, complications such as VAP and CAUTI were more frequently observed among patients with a PLS (log-rank $P=0.005$ and 0.048 , respectively). By contrast, no significant differences in CRBSI $(P=0.33)$ and CPR in the ICU $(P=0.52)$ were observed between patients in the PLS and non-PLS groups.

\section{Discussion}

In this study of $>1300$ patients admitted to a single PICU for diverse reasons, we demonstrated the significant effects of long-stay patients on PICU bed utilization and resource consumption and revealed the factors associated with a PLS in this population. Notably, although only $2.4 \%$ of patients had an LOS $\geq 28$ days, this group accounted for $33 \%$ of all PICU bed days during the study period. Additionally, approximately 
Table 1 Comparison of demographics and characteristics between PLS and non-PLS groups

\begin{tabular}{|c|c|c|c|c|c|}
\hline Variable & All $n=1309$ & PLS $n=32$ & Non-PLS $n=1277$ & Odds ratio $[95 \% \mathrm{CI}]$ & $P$ value \\
\hline \multicolumn{6}{|l|}{ Age } \\
\hline Neonate $^{\mathrm{a}}$ & $101(7 \%)$ & $9(28 \%)$ & $92(7 \%)$ & $7.8[2.5-25.4]$ & $<0.001$ \\
\hline Infant & $240(18 \%)$ & $11(34 \%)$ & $229(18 \%)$ & $2.9[1.0-9.0]$ & 0.04 \\
\hline $1-6$ years & $558(43 \%)$ & $5(16 \%)$ & $553(43 \%)$ & $0.5[0.1-2.0]$ & 0.38 \\
\hline$>6$ years & $410(31 \%)$ & $7(22 \%)$ & $403(32 \%)$ & Reference & - \\
\hline Female & $577(44 \%)$ & $10(31 \%)$ & $567(44 \%)$ & $0.6[0.2-1.2]$ & 0.15 \\
\hline \multicolumn{6}{|l|}{ Primary reason for admission } \\
\hline Respiratory & $149(11 \%)$ & $7(21 \%)$ & $142(11 \%)$ & $7.3[1.6-44.2]$ & 0.003 \\
\hline Cardiovascular & $43(6 \%)$ & $6(19 \%)$ & $37(3 \%)$ & $24.1[4.8-152.1]$ & $<0.001$ \\
\hline Neurologic & $176(13 \%)$ & $4(13 \%)$ & $172(13 \%)$ & $3.4[0.6-23.7]$ & 0.10 \\
\hline PCAS & $15(1 \%)$ & $2(6 \%)$ & $13(1 \%)$ & $22.8[1.7-211.9]$ & 0.01 \\
\hline Trauma & $54(4 \%)$ & $2(6 \%)$ & $52(4 \%)$ & $5.7[0.5-50.7]$ & 0.09 \\
\hline Other & $307(23 \%)$ & $2(6 \%)$ & $305(24 \%)$ & $0.97[0.1-8.5]$ & 1.00 \\
\hline Surgical cardiac & $117(9 \%)$ & $6(19 \%)$ & $111(7 \%)$ & $8.0[1.7-50.1]$ & 0.003 \\
\hline Surgical non-cardiac & $448(34 \%)$ & $3(9 \%)$ & $445(35 \%)$ & Reference & - \\
\hline \multicolumn{6}{|l|}{ Origin of admission } \\
\hline Emergency room & $385(29 \%)$ & $3(9 \%)$ & $382(30 \%)$ & $0.5[0.1-2.1]$ & 0.38 \\
\hline Transfer & $299(23 \%)$ & $18(56 \%)$ & $281(22 \%)$ & $4.2[1.8-10.7]$ & 0.001 \\
\hline Ward & $29(2 \%)$ & $2(6 \%)$ & $27(2 \%)$ & $4.8[0.5-24.9]$ & 0.09 \\
\hline Operation room & $596(46 \%)$ & $9(28 \%)$ & $587(46 \%)$ & Reference & - \\
\hline \multicolumn{6}{|l|}{ Patient characteristic } \\
\hline Chromosomal abnormality & $102(8 \%)$ & $5(16 \%)$ & $97(8 \%)$ & $2.3[0.7-6.1]$ & 0.10 \\
\hline Baseline $\mathrm{PCPC} \geq 2$ & $300(23 \%)$ & $14(44 \%)$ & $286(22 \%)$ & $2.7[1.2-5.8]$ & 0.01 \\
\hline Comorbidities $\geq 2^{\mathrm{b}}$ & $233(18 \%)$ & $11(34 \%)$ & $222(17 \%)$ & $2.8[1.1-6.9]$ & 0.03 \\
\hline Comorbidity $^{\mathrm{c}}$ & $397(30 \%)$ & $9(28 \%)$ & $388(30 \%)$ & $1.3[0.5-3.4]$ & 0.65 \\
\hline No comorbidities & $679(52 \%)$ & $12(38 \%)$ & $667(52 \%)$ & Reference & - \\
\hline Tube feeding ${ }^{\mathrm{d}}$ & $113(9 \%)$ & $8(25 \%)$ & $105(8 \%)$ & $3.7[1.4-8.8]$ & 0.004 \\
\hline $\mathrm{TPN}^{\mathrm{e}}$ & $6(0.5 \%)$ & 0 & $6(0.5 \%)$ & - & 1.00 \\
\hline Chronic ventilation ${ }^{\mathrm{f}}$ & $39(3 \%)$ & $2(6 \%)$ & $37(3 \%)$ & $2.2[0.2-9.4]$ & 0.25 \\
\hline Emergent admission & $746(57 \%)$ & $24(75 \%)$ & $722(57 \%)$ & $2.3[1.0-6.0]$ & 0.046 \\
\hline MV within $1 \mathrm{~h}$ & $247(19 \%)$ & $26(81 \%)$ & $221(17 \%)$ & $20.7[8.2-62.0]$ & $<0.001$ \\
\hline PIM-2 & $2.2(9.2)$ & $7.3(13 \%)$ & $2.0(9 \%)$ & - & $<0.001$ \\
\hline
\end{tabular}

PLS, prolonged length of stay; $n$, number; $\mathrm{CI}$, confidence interval; $P$ value, probability of finding; PCAS, post-cardiopulmonary arrest; PCPC, pediatric cerebral performance category; TPN, total parenteral nutrition; MV, mechanical ventilation; PIM, pediatric index of mortality

Values provided as numbers (percentages) for categorical variables and as means (standard deviations) for continuous variables

Odds ratios provided with $95 \%$ confidence intervals

${ }^{\text {a }}$ Patients admitted at $\leq 30$ days of age

${ }^{\mathrm{b}}$ Patients with more than two comorbidities

${ }^{\mathrm{c}}$ Patients with one comorbidity

d, e, f Chronic dependency on tube feeding, total parenteral nutrition, or respiratory support

half of all PICU bed days were used by the $5.9 \%$ of patients with an LOS $>14$ days.

Although a longitudinal study conducted in Australia reported a PLS prevalence of $1 \%$ in PICU settings, this prevalence had increased three-fold during the study period 19892008, suggesting an alarming increase in this patient population (Namachivayam et al. 2012). In other studies published between 2009 and 2011, the prevalence of patients with PICU LOS $\geq 28$ days ranged from $3.1 \%$ to $4.4 \%$ (Conlon et al. 2009; Gonzalez-Cortes et al. 2011; Naghib et al. 2010). We noted in our study that the prevalence of PLS remained substantial despite our efforts to reduce LOS by providing fast-track care. These findings from our own and previous studies indicate that long-stay patients may present challenges that cannot be 


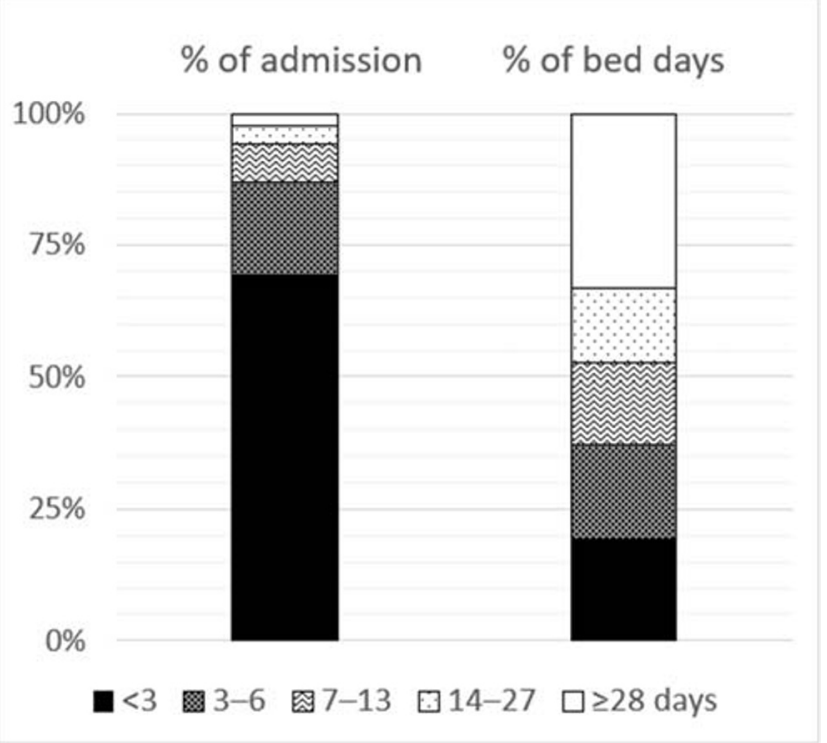

Fig. 1 Pediatric intensive care unit (PICU) utilization by percentage of admissions (left) bed days (right). Note that $2.4 \%$ of patients with a length of stay (LOS) $\geq 28$ days accounted for $33.0 \%$ of the overall ICU bed days. The combined $5.9 \%$ of patients with an LOS $\geq 28$ or $14-27$ days accounted for $47 \%$ of bed days, whereas the $69.4 \%$ of patients with an LOS of $<3$ days and $17.7 \%$ of patients with an LOS of 3-6 days accounted for $19.3 \%$ and $17.9 \%$ of ICU bed days, respectively.

alleviated by such a practice, which might suggest the need for further efforts to reduce LOS by implementing quality improvement programs such as the Awakening and Breathing Coordination, Delirium Monitoring/Management, and Early Exercise/Mobility (ABCDE) bundle (Balas et al. 2013; McWilliams et al. 2015). However, the efficacy of such programs will need to be determined in future studies.

In our study, age was identified as an influential factor regarding PLS, consistent with previous studies. Patients requiring medical or surgical treatments during the early stage of life may be more likely to suffer from severe diseases and comorbidities (Pollack et al. 2018; Marcin et al. 2001). In addition, young children often require prolonged recovery time (Mori et al. 2016). We further observed that patients with a PLS were commonly admitted with cardiorespiratory dysfunction and post-cardiopulmonary arrest. Patients with organ dysfunction may be critically ill, and the required advanced treatments could lead to a PLS (Pollack et al. 2018). We also found that postsurgical cardiac patients were at risk of a PLS, although two thirds of cardiac patients $>1$ year old had been extubated in the OR. Although it remains controversial whether cardiac patients are at risk of PLS, two studies reported a particularly high likelihood of PLS among neonates who underwent cardiac surgery (Mori et al. 2016; Namachivayam et al. 2012). Our results might also be explained by the fact that $20 \%$ of children in our study who underwent cardiac surgery were $<1$ month old.
Table 2 Comparison of resource consumption and complications between PLS and non-PLS groups

\begin{tabular}{lllll}
\hline Variable & $\begin{array}{l}\text { All } \\
\mathrm{n}=1309\end{array}$ & $\begin{array}{l}\mathrm{PLS} \\
\mathrm{n}=32\end{array}$ & $\begin{array}{l}\text { Non-PLS } \\
\mathrm{n}=1277\end{array}$ & $P$ value \\
\hline Device & & & & \\
$\quad$ Arterial line & $309(24 \%)$ & $26(81 \%)$ & $283(22 \%)$ & $<0.001$ \\
CV line & $194(15 \%)$ & $21(66 \%)$ & $173(14 \%)$ & $<0.001$ \\
Foley catheter & $486(37 \%)$ & $28(88 \%)$ & $458(36 \%)$ & $<0.001$ \\
Therapeutic resource & & & & \\
MV & $260(20 \%)$ & $26(81 \%)$ & $234(18 \%)$ & $<0.001$ \\
Vasoactive drug & $151(12 \%)$ & $21(66 \%)$ & $130(10 \%)$ & $<0.001$ \\
RRT & $17(1 \%)$ & $7(22 \%)$ & $10(1 \%)$ & $<0.001$ \\
ECMO & $3(0.2 \%)$ & $2(6 \%)$ & $1(0.1 \%)$ & 0.002 \\
Surgical intervention & & & & \\
Tracheostomy & $7(0.5 \%)$ & $5(16 \%)$ & $2(0.2 \%)$ & $<0.001$ \\
Gastrostomy & $1(0.1 \%)$ & $1(3 \%)$ & 0 & 0.02 \\
Complication and outcome & & & \\
CRBSI & $6(0.5 \%)$ & $4(13 \%)$ & $2(0.2 \%)$ & $0.33^{*}$ \\
VAP & $8(1 \%)$ & $6(19 \%)$ & $2(0.2 \%)$ & $0.005^{*}$ \\
CAUTI & $11(1 \%)$ & $6(19 \%)$ & $5(0.4 \%)$ & $0.048^{*}$ \\
CPR in the ICU & $4(0.3 \%)$ & 0 & $4(0.3 \%)$ & $0.52^{*}$ \\
Mortality & $17(1.2 \%)$ & $3(9 \%)$ & $14(1 \%)$ & - \\
\hline
\end{tabular}

PLS, prolonged length of stay; $n$, number; $P$ value, probability of finding; $\mathrm{CV}$, central venous; $\mathrm{MV}$, mechanical ventilation; RRT, renal replacement therapy; ECMO, extracorporeal membrane oxygenation; CRBSI, catheter-related bloodstream infection; VAP, ventilator-associated pneumonia; CAUTI, catheter-associated urinary tract infection; CPR, cardiopulmonary resuscitation; ICU, intensive care unit

Values provided as numbers (percentages) for categorical variables

${ }^{*} P$ values for time-dependent variables calculated using log-rank test

Regarding the origin of admission, we found that patients transferred from other hospitals had a high likelihood of PLS, consistent with previous research (Straney et al. 2010). We note that in our study, half of the referred patients presented with dysfunction in at least one organ, and some required RRT and ECMO. However, since patient transportation also appears to correlate with other patient-related risk factors for PLS, we cannot conclude whether such transfer is an intrinsically influential factor. We further note that admission from another ward was not significantly associated with PLS in our study. However, the limited number of patients in this category might have attenuated a potential association between this type of admission and PLS. Therefore, we cannot draw a conclusion about this link at present.

Consistent with our findings, reports in the literature have demonstrated the association between the Pediatric Risk of Mortality (PRISM) score and LOS, except among patients with a PRISM score of $>33$ (Marcin et al. 2001; Pollack et al. 2018). As in our study, previous studies have also identified PLS linkage with other factors, such as emergent 


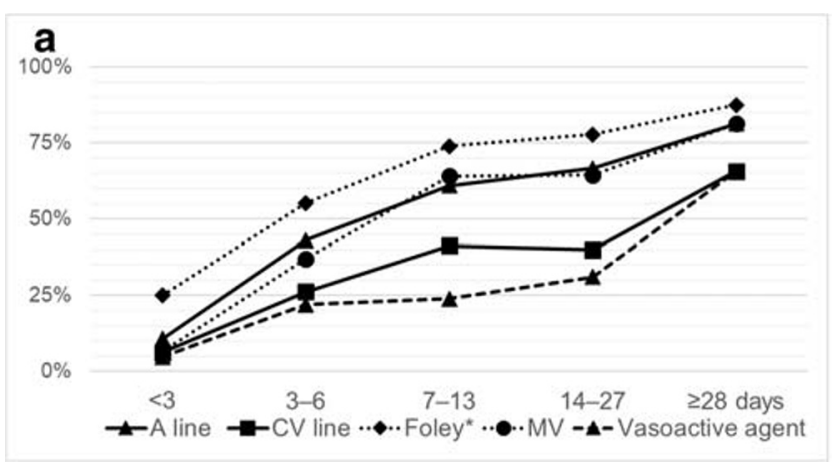

Fig. 2 Frequencies of device, treatment, and surgical intervention usage among patients stratified by length of stay. The frequencies of utilization in categories such as arterial line, central venous line, Foley catheter, vasoactive drug, and mechanical ventilation increased as the length of stay was prolonged (a). However, RRT, ECMO, tracheostomy, and

admission, comorbidities, high PCPC, dependency on tube feeding, and MV within the first hour of admission (Straney et al. 2010; Marcin et al. 2001; Pollack et al. 2018; Fiser et al. 2000). However, the lack of a correlation between dependency on chronic respiratory support and PLS in our study contradicts the identification of such dependency as a risk factor for PLS in a previous study (Marcin et al. 2001). This discrepancy may be attributed to the low threshold of ICU admission for those with underlying respiratory diseases, with such patients being admitted to the ICU with relatively less severe respiratory dysfunction.

Regarding device usage, we found that most devices included in our analysis were commonly applied to patients with a PLS. Specifically, our descriptive analyses revealed an increasing trend between a longer LOS and the frequency of use of devices such as an arterial line, Foley catheter, central venous line, vasoactive drug, or MV. We further observed that ECMO, RRT, tracheostomy, and gastrostomy were mostly

(day)

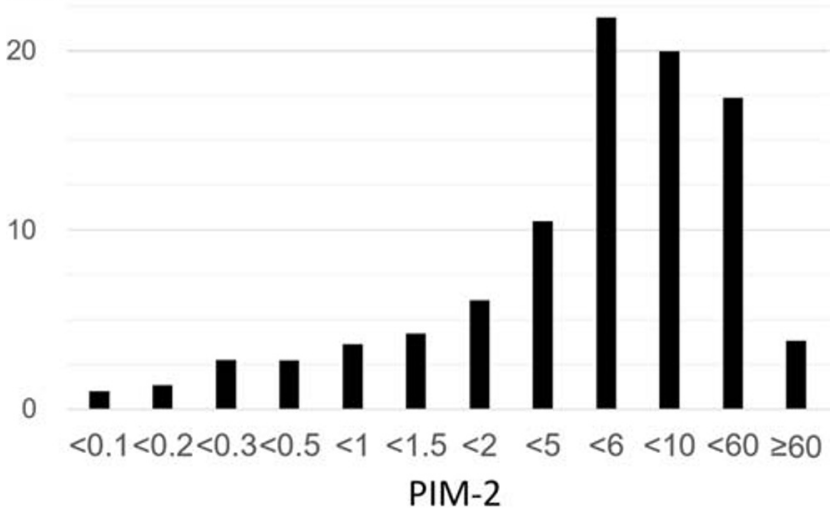

Fig. 3 Mean length of stay according to PIM-2 (pediatric risk of mortality) category. A positive correlation was observed between a PIM-2 of 0 $6 \%$ and the length of stay, whereas the length of stay decreased at higher PIM-2 values

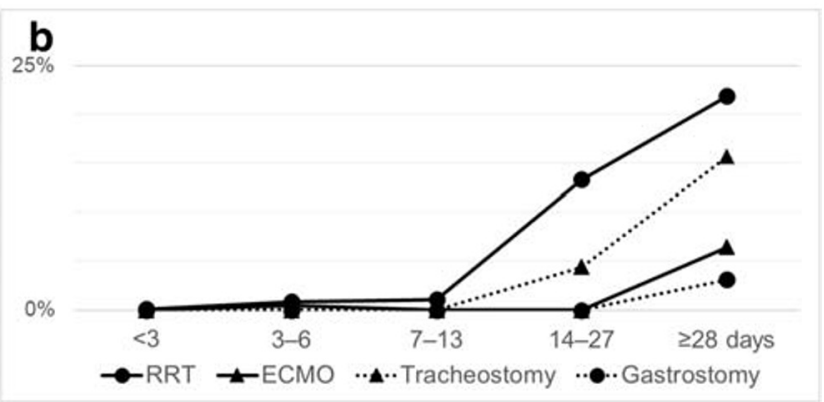

gastrostomy were predominantly applied to patients with a length of stay $\geq 14$ days (b). A line, arterial line; CV line, central venous line; $\mathrm{MV}$, mechanical ventilation; RRT, renal replacement therapy; ECMO, extracorporeal membrane oxygenation. Foley*: Foley catheter

applied to patients with an LOS $\geq 14$ days, suggesting that these patients may have a different clinical course relative to those with an $\operatorname{LOS}<14$ days. Future studies should determine whether 14 or 28 days is a reasonable definition of a PLS.

We further found that VAP and CAUTI, but not CRBSI, were associated with PLS. Although previous studies have reported that patients who developed nosocomial infections have relatively longer ICU LOS (Barnett et al. 2013; Karvouniaris et al. 2013), few have compared the incidence of nosocomial infections between PLS and nonPLS patients. Therefore, our finding suggests the need for further research to avoid infectious complications among long-stay patients.

\section{Limitations}

Our study had several limitations of note. First, it was conducted at a single institution, which may limit the generalizability of our findings to other institutions. However, a contemporary multicenter study conducted in the USA reported a PLS prevalence of $2.3 \%$, which is strikingly similar to our finding (Pollack et al. 2018). Therefore, we believe that our findings may provide a reasonable approximation for settings in developed countries. Second, the limited sample size underpowered our risk factor analysis. As we expected limited cases with PLS a priori, we decided to focus on descriptive rather than multivariable analysis. Consequently, we could not draw conclusions regarding the correlations and modifications among the possible risk factors for PLS. Third, the study outcomes may have been influenced by institutional practice and physician' preferences, given the considerable variability in decisions regarding discharge from the PICU. As with previous studies, we could not identify the criteria determining admission to and discharge from the PICU. Future studies are needed to determine the indications for a PICU stay. 


\section{Conclusions}

This study found that long-stay patients disproportionately utilize the PICU and intensive care resources. Furthermore, we identified age $<1$ year, cardiorespiratory dysfunction, post-surgical cardiac patient status, and transfer from another hospital as possible risk factors for a PLS. So-called "complicated" patients are those at risk of a PSL in a tertiary intensive care unit. Our findings warrant further studies in tertiary centers, with the aim of reducing institutional and patient-related issues in the affected population.

Acknowledgments All authors contributed to the research concept/idea/ design, data collection, writing, and data analysis. Dr. Shinya provided project management and data management.
Funding sources This study was supported by the Saitama Children's Medical Center Research Fund. This research is funded by Saitama Children's Medical Center research fund. Funding was only used to support submission and publication. All authors report no other potentially relevant conflicts of interest.

\section{Compliance with ethical standards}

Conflict of interest The authors declare that they have no conflict of interest.

Ethical approval This study was approved by the institutional review board (IRB) at the Saitama Children's Medical Center, and the need for informed consent was waived. This study was performed in accordance with the Declaration of Helsinki.

\section{Appendix}

Fig. 4 Percentages of patients in five length-of-stay categories, stratified by age. Neonates comprised a considerable proportion of patients with a prolonged length of stay, whereas only a few pre-school and school-aged patients had stays $>14$ days
Fig. 5 Percentages of patients in five length-of-stay categories, stratified by admission category. Patients admitted for respiratory or cardiovascular dysfunction, post-cardiac arrest, and cardiac surgery had substantially longer lengths of stay than non-cardiac surgical patients. PCAS, postcardiopulmonary arrest
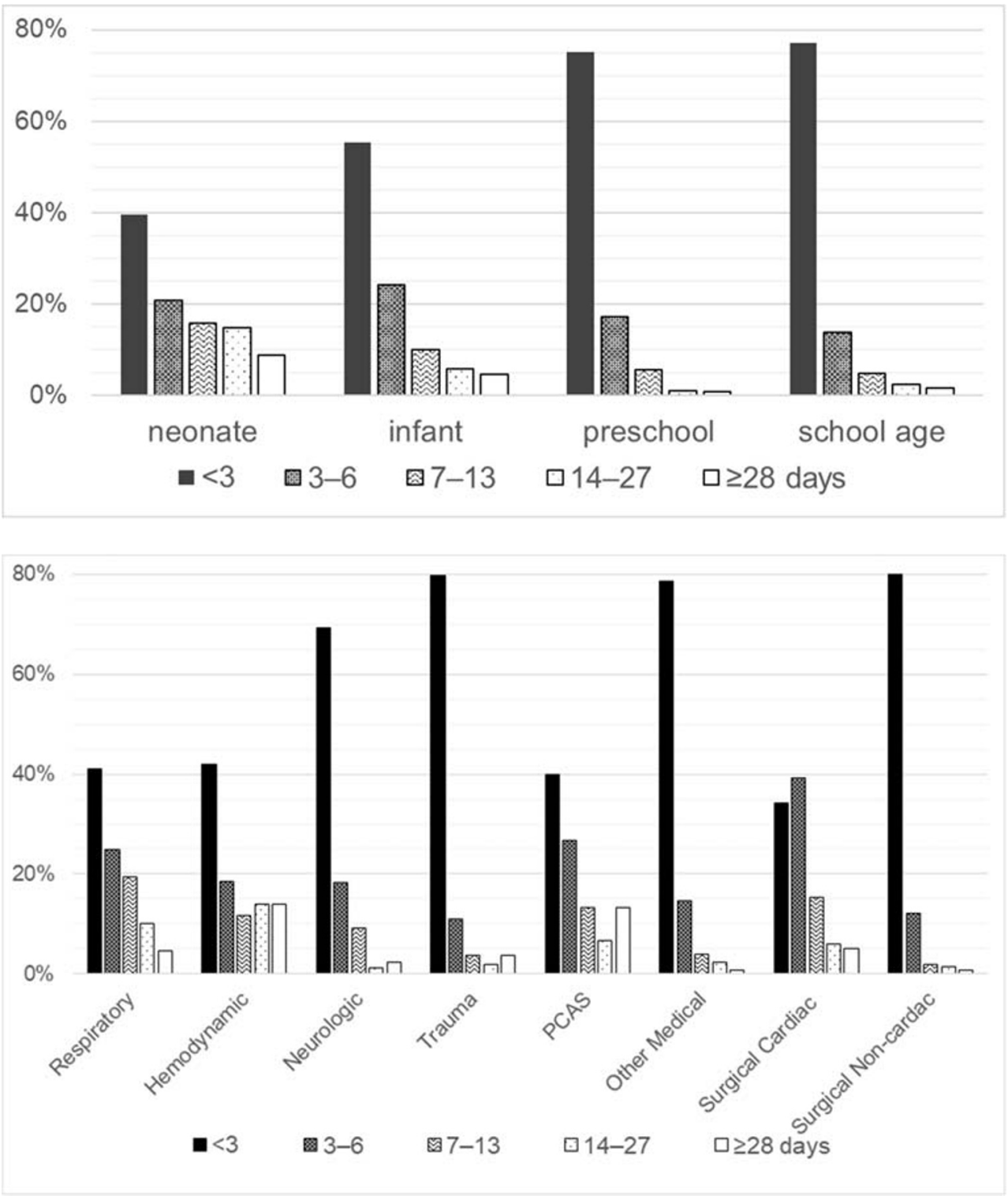
Fig. 6 Percentages of patients in five length-of-stay categories, stratified by origin of admission. Although the majority of patients admitted from the operation room and emergency room stayed for $<$ 7 days, a considerable percentage of patients transferred from other hospitals and wards stayed for $\geq$ 14 days. ER, emergency room; $\mathrm{OR}$, operation room

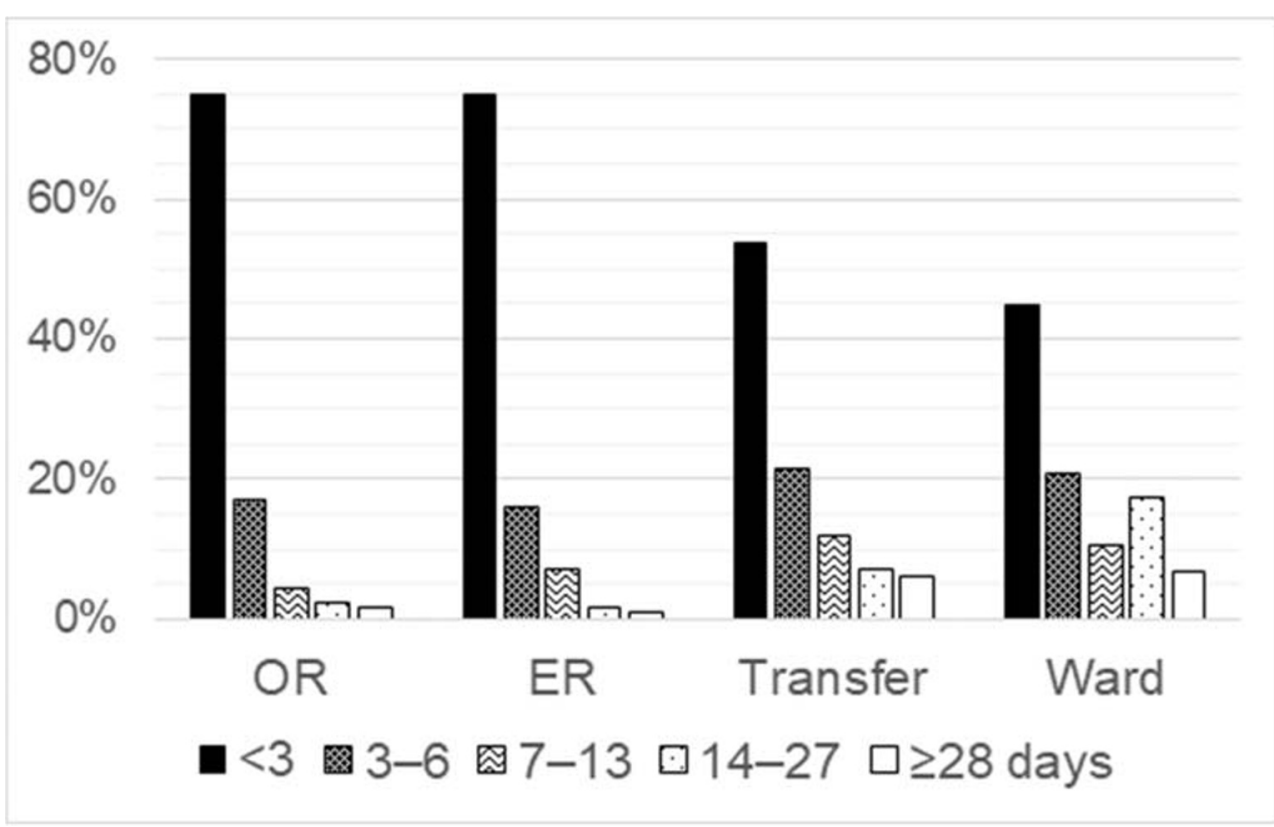

\section{References}

Balas MC, Burke WJ, Gannon D, Cohen MZ, Colburn L, Bevil C, Franz D, Olsen KM, Ely EW, Vasilevskis EE (2013) Implementing the awakening and breathing coordination, delirium monitoring/management, and early exercise/mobility bundle into everyday care: opportunities, challenges, and lessons learned for implementing the ICU pain, agitation, and delirium guidelines. Crit Care Med 41(9 Suppl 1):S116-S127

Barnett AG, Page K, Campbell M, Martin E, Rashleigh-Rolls R, Halton K, Paterson DL, Hall L, Jimmieson N, White K, Graves N (2013) The increased risks of death and extra lengths of hospital and ICU stay from hospital-acquired bloodstream infections: a case-control study. BMJ Open 3(10):e003587

Conlon NP, Breatnach C, O'Hare BP, Mannion DW, Lyons BJ (2009) Health-related quality of life after prolonged pediatric intensive care unit stay. Pediatr Crit Care Med 10(1):41-44

da Silva PS, Reis ME, Fonseca TS, Fonseca MC (2016) Do in-hours or off-hours matter for extubating children in the pediatric intensive care unit? J Crit Care 36:97-101

Dreyfus L, Javouhey E, Denis A, Touzet S, Bordet F (2017) Implementation and evaluation of a paediatric nurse-driven sedation protocol in a paediatric intensive care unit. Ann Intensive Care 7(1): 36

Fiser DH, Tilford JM, Roberson PK (2000) Relationship of illness severity and length of stay to functional outcomes in the pediatric intensive care unit: a multi-institutional study. Crit Care Med 28(4):11731179

González-Cortés R, López-Herce-Cid J, García-Figueruelo A, TesoreroCarcedo G, Botrán-Prieto M, Carrillo-Álvarez A (2011) Prolonged stay in pediatric intensive care units: mortality and healthcare resource consumption. Med Int 35(7):417-423

Johnson JT, Wilkes JF, Menon SC, Tani LY, Weng HY, Marino BS, Pinto NM (2018) Admission to dedicated pediatric cardiac intensive care units is associated with decreased resource use in neonatal cardiac surgery. J Thorac Cardiovasc Surg 155(6):2606-2614.e5

Karvouniaris M, Makris D, Manoulakas E, Zygoulis P, Mantzarlis K, Triantaris A, Chatzi M, Zakynthinos E (2013) Ventilatorassociated tracheobronchitis increases the length of intensive care unit stay. Infect Control Hosp Epidemiol 34(8):800-808

Mahesh B, Choong CK, Goldsmith K, Gerrard C, Nashef SA, Vuylsteke A (2012) Prolonged stay in intensive care unit is a powerful predictor of adverse outcomes after cardiac operations. Ann Thorac Surg 94(1):109-116

Marcin JP, Slonim AD, Pollack MM, Ruttimann UE (2001) Long-stay patients in the pediatric intensive care unit. Crit Care Med 29(3): 652-657

McWilliams D, Weblin J, Atkins G, Bion J, Williams J, Elliott C, Whitehouse T, Snelson C (2015) Enhancing rehabilitation of mechanically ventilated patients in the intensive care unit: a quality improvement project. J Crit Care 30(1):13-18

Mori M, McCracken C, Maher K, Kogon B, Mahle W, Kanter K, Alsoufi B (2016) Outcomes of neonates requiring prolonged stay in the intensive care unit after surgical repair of congenital heart disease. J Thorac Cardiovasc Surg 152(3):720-727.e1

Naghib S, van der Starre C, Gischler SJ, Joosten KF, Tibboel D (2010) Mortality in very long-stay pediatric intensive care unit patients and incidence of withdrawal of treatment. Intensive Care Med 36(1): $131-136$

Namachivayam P, Taylor A, Montague T, Moran K, Barrie J, Delzoppo C, Butt W (2012) Long-stay children in intensive care: long-term functional outcome and quality of life from a 20-yr institutional study. Pediatr Crit Care Med 13(5):520-528

Pollack MM, Holubkov R, Reeder R, Dean JM, Meert KL, Berg RA, CJL N, Berger JT, Harrison RE, Carcillo J, Dalton H, Wessel DL, Jenkins TL, Tamburro R, Eunice Kennedy Shriver National Institute of Child Health and Human Development Collaborative Pediatric Critical Care Research Network (CPCCRN) (2018) PICU length 
of stay: factors associated with bed utilization and development of a benchmarking model. Pediatr Crit Care Med 19(3):196-203

Pollack MM, Wilkinson JD, Glass NL (1987) Long-stay pediatric intensive care unit patients: outcome and resource utilization. Pediatrics 80(6):855-860

Reismann M, Arar M, Hofmann A, Schukfeh N, Ure B (2012) Feasibility of fast-track elements in pediatric surgery. Eur J Pediatr Surg 22(1): 40-44

Riker RR, Shehabi Y, Bokesch PM, Ceraso D, Wisemandle W, Koura F, Whitten P, Margolis BD, Byrne DW, Ely EW, Rocha MG, SEDCOM (Safety and Efficacy of Dexmedetomidine Compared With Midazolam) Study Group (2009) Dexmedetomidine vs midazolam for sedation of critically ill patients: a randomized trial. Jama 301(5):489-499

Ruttimann UE, Patel KM, Pollack MM (2000) Relevance of diagnostic diversity and patient volumes for quality and length of stay in pediatric intensive care units. Pediatr Crit Care Med 1(2):133-139

Ruttimann UE, Pollack MM (1996) Variability in duration of stay in pediatric intensive care units: a multiinstitutional study. J Pediatr 128(1):35-44
Shann F, Pearson G, Slater A, Wilkinson K (1997) Paediatric index of mortality (PIM): a mortality prediction model for children in intensive care. Intensive Care Med 23(2):201-207

Straney L, Clements A, Alexander J, Slater A; ANZICS Paediatric Study Group (2010) Quantifying variation of paediatric length of stay among intensive care units in Australia and New Zealand. Qual Saf Health Care 19 (6): e5

Tischenkel BR, Gong MN, Shiloh AL, Pittignano VC, Keschner YG, Glueck JA, Cohen HW, Eisen LA (2016) Daytime versus nighttime extubations: a comparison of reintubation, length of stay, and mortality. J Intensive Care Med 31(2):118-126

Vricella LA, Dearani JA, Gundry SR, Razzouk AJ, Brauer SD, Bailey LL (2000) Ultra fast track in elective congenital cardiac surgery. Ann Thorac Surg 69(3):865-871

Yamasaki Y, Shime N, Miyazaki T, Yamagishi M, Hashimoto S, Tanaka Y (2011) Fast-track postoperative care for neonatal cardiac surgery: a single-institute experience. J Anesth 25(3):321-329

Publisher's note Springer Nature remains neutral with regard to jurisdictional claims in published maps and institutional affiliations. 